\title{
Efficient and specific repair of sickle $\beta$-globin RNA by trans-splicing ribozymes
}

\author{
JONGHOE BYUN, NING LAN, MEREDITH LONG, and BRUCE A. SULLENGER \\ Center for Genetic and Cellular Therapies, Departments of Surgery and Genetics, Duke University Medical Center, \\ Durham, North Carolina 27710, USA
}

\begin{abstract}
Previously we demonstrated that a group I ribozyme can perform trans-splicing to repair sickle $\beta$-globin transcripts upon transfection of in vitro transcribed ribozyme into mammalian cells. Here, we sought to develop expression cassettes that would yield high levels of active ribozyme after gene transfer. Our initial expression constructs were designed to generate trans-slicing ribozymes identical to those used in our previous RNA transfection studies with ribozymes containing 6-nucleotide long internal guide sequences. The ribozymes expressed from these cassettes, however, were found to be unable to repair sickle $\beta$-globin RNAs. Further experiments revealed that two additional structural elements are important for ribozyme-mediate RNA repair: the P10 interaction formed between the 5' end of the ribozyme and the beginning of the $3^{\prime}$ exon and an additional base-pairing interaction formed between an extended guide sequence and the substrate RNA. These optimized expression cassettes yield ribozymes that are able to amend $\mathbf{1 0} \%-\mathbf{5 0} \%$ of the sickle $\beta$-globin RNAs in transfected mammalian cells. Finally, a ribozyme with a 5-bp extended guide sequence preferentially reacts with sickle $\beta$-globin RNAs over wild-type $\beta$-globin RNAs, although the wild-type $\beta$-globin transcript forms only a single mismatch with the ribozyme. These results demonstrate that trans-splicing ribozyme expression cassettes can be generated to yield ribozymes that can repair a clinically relevant fraction of sickle $\beta$-globin RNAs in mammalian cells with greatly improved specificity.
\end{abstract}

Keywords: Trans-splicing; ribozyme; RNA repair; sickle $\beta$-globin; specificity; efficiency

\section{INTRODUCTION}

Trans-splicing ribozymes can be used to amend defective transcripts for therapeutic purposes (Sullenger and Cech 1994; Phylactou et al. 1998; Lan et al. 1998). Previously, we have demonstrated that the trans-splicing ribozyme from the Tetrahymena group I intron can be transfected into erythrocyte precursor cells to convert $\beta^{\mathrm{s}}$-globin transcripts into mRNAs encoding the anti-sickling $\gamma$-globin protein (Lan et al. 1998). For therapeutic use, however, an expression system must be established that will produce catalytically active ribozymes inside the target cells of patients. Development of efficient and stable in vivo expression systems for the Tetrahymena ribozymes is, therefore, a pressing issue for trans-splicing ribozyme studies and their future development.

One of the unique challenges for constructing ribozyme expression cassettes is to design transcription units with

Reprint requests to: Bruce A. Sullenger, Center for Genetic and Cellular Therapies, Departments of Surgery and Genetics, Duke University Medical Center, Durham, NC 27710, USA; e-mail: b.sullenger@cgct.duke.edu.

Article and publication are at http://www.rnajournal.org/cgi/doi/ 10.1261/rna.5450203. necessary structural elements that do not significantly inhibit the ribozyme's catalytic activity (Couture and Stinchcomb 1996). Furthermore, a highly efficient expression system may be necessary to transcribe an excess of ribozyme molecules over target transcripts because ribozyme-mediated trans-splicing is a single turnover reaction. To express ribozymes, RNA polymerase II (pol II) and III (pol III) promoters have been used. RNA pol III-based expression cassettes, containing the tRNA, U6, and adenoviral VA1 promoters, have been extensively used to express small RNAs such as antisense RNAs (Jennings and Molloy 1987; Sullenger et al. 1990a), trans-cleaving ribozymes (Ojwang et al. 1992), RNA decoys (Sullenger et al. 1990b), and siRNAs (Lee et al. 2002; Miyagishi and Taira 2002; Paul et al. 2002; Sui et al. 2002). RNA pol III transcripts accumulate to high levels in transduced mammalian cells. However, efficient expression of long RNAs by RNA pol III promoters has been difficult to achieve (B. Sullenger, unpubl.). The Tetrahymena group I ribozyme is $\sim 400$ nucleotides in length and the therapeutic $3^{\prime}$ exon sequences attached to the ribozyme can be thousands of nucleotides in length, rendering a pol III-based system less suitable for trans-splicing ribozyme expression. Therefore, in these studies we focused on the development of pol II-based expression cassettes. 
A detailed understanding of the self- and trans-splicing reactions catalyzed by the Tetrahymena group I intron has been established. Within the catalytic core of the intron, the intron-exon junctions are aligned along the internal guide sequence (IGS) forming a 9-bp helix, termed P1, during the first step of self-splicing (Been and Cech 1986; Waring et al. 1986; Michel et al. 1989). After cleavage, part of the P1 helix is displaced to allow formation of a new helix, termed P10, based on complementarity between the IGS and sequences in the $3^{\prime}$ exon adjacent to the $3^{\prime}$ splice site. Although the P10 interaction within the Tetrahymena intron is not essential to the recognition of the $3^{\prime}$ site in vitro (Been and Cech 1986), it appears to increase the efficiency and specificity of splicing, and is conserved in most group I introns (Michel and Westhof 1990; Suh and Waring 1990; Lehnert et al. 1996). Haseloff and colleagues have shown that by extending the P1 helix from 6 to 9 nucleotides, attaching the P10 helix and adding an extended base-pairing interaction of 45-200 nucleotides complementary to the intended target RNA then the specificity and efficiency of ribozyme-mediated trans-splicing could be increased in bacteria (Kohler et al. 1999). More recently our group demonstrated that a transsplicing ribozyme containing an extended guide sequence (EGS) and the P10 interaction is able to repair mutant p53 transcripts in human cancer cells (Watanabe and Sullenger 2000).

In this study, we sought to optimize the efficiency and specificity of trans-splicing ribozymes expressed by RNA pol II in mammalian cells. We systematically analyzed the importance of the P10 helix, antisense complementarity, and different promoters on the efficiency and targeting specificity of the trans-splicing ribozyme. Our results provide some insight into the logical design of ribozyme expression cassettes that confer improved efficiency and specificity. Also they are surprising in that they suggest that transsplicing ribozymes may be much more specific for their target RNAs than previously suspected. Finally, they demonstrate that trans-splicing ribozymes can revise a clinically relevant fraction of sickle $\beta$-globin RNA.

\section{RESULTS}

\section{Pol II expressed trans-splicing ribozymes require additional sequence elements}

Previously we demonstrated that the Rib61-3' $\gamma$ ribozyme could repair sickle $\beta$-globin RNA when directly transfected into mammalian cells (Lan et al. 1998, 2000). Rib61-3' $\gamma$ targets the uridine at position 61 of the $\beta^{\mathrm{s}}$-globin mRNA and trans-splices a $3^{\prime}$ exon containing the wild-type $\gamma$-globin sequence onto the targeted uridine. To determine whether this same ribozyme could repair sickle $\beta$-globin transcripts after gene transfer and expression, we cloned Rib61-3' $\gamma$ into a mammalian expression vector. This construct, termed S0, contains the Cytomegalovis (CMV) im- mediate early promoter and the SV40 polyadenylation signal and is designed to express the Rib61-3' $\gamma$ ribozyme with a 6-nucleotide long IGS (Fig. 1A). To evaluate the activity of the ribozyme expressed from the S0 construct, we cotransfected HEK293 cells with the S0-containing plasmid and a $\beta^{\mathrm{s}}$-globin substrate plasmid (pNL97) that harbors the genomic version of the sickle $\beta$-globin gene under the control of the CMV promoter. Total RNA was isolated from transfected cells and analyzed for the presence of trans-spliced products using reverse transcription and PCR (RT/PCR) amplification. Surprisingly, and in contrast to our previous results with direct RNA transfection, no trans-spliced products were detected (Fig. 1C, lane 5) by even this sensitive assay, although both the ribozyme and $\beta^{\text {s}}$-globin substrate were present in the cells (data not shown).

To determine whether additional sequence elements were required to yield an active version of the Rib61-3' $\gamma$ after RNA pol II expression, we generated and evaluated a series of modified ribozyme constructs. Because the $5^{\prime}$ end of pol II transcripts are capped and are bound by a large polyprotein cap binding complex (Shatkin 1985), we generated three new ribozyme constructs to determine whether moving the ribozyme further from the $5^{\prime}$ end of the RNA transcript would facilitate trans-splicing. The S1, S3, and S5 ribozyme derivatives were created by inserting 41 additional nucleotides on the $5^{\prime}$ end of the S0 ribozyme. In S1, these nucleotides are complementary to $\beta^{\text {s}}$-globin RNA (nucleotides 62-102) and can act as an EGS that form a 47-nucleotide long duplex with the $\beta^{\text {s }}$-globin target RNA (Fig. 1A). The S3 construct contains a 41-nucleotide long $5^{\prime}$ spacer sequence that is unlikely to form base-pairs with the $\beta^{s}$ globin target RNA (Fig. 1A). The S5 derivative contains an extended P1 helix (9 nucleotides), the P10 helix (6 nucleotides), and an additional 35-nucleotide long EGS (EGS) complementary to $\beta^{\mathrm{s}}$-globin RNA (Fig. 1A). Thus, the S5 riboyzme differs from $\mathrm{S} 1$ in that $\mathrm{S} 5$ contains the P10 interaction, which interrupts the long duplex formed between the ribozyme and the substrate RNA present in S1 (Fig. 1B). To evaluate the activity of these ribozyme derivatives, HEK293 cells were cotransfected with ribozyme and substrate expression plasmids and total cellular RNA was isolated and evaluated for trans-splicing products. As shown in Figure 1C, only RNA from cells transfected with the S5 construct contained detectable amounts of repaired $\beta^{\text {s}}$-globin RNA (lane 2) indicating that catalytically active transsplicing ribozymes can be expressed in mammalian cells. No amplification products were generated from the controls that were not transfected (lane 9), transfected with $\beta^{\mathrm{s}}$-globin substrate plasmid pNL97 alone (lane 8), cotransfected with inactive ribozyme (lane 7), or in the mix control (lane 6) where separately transfected HEK293 cells (S5transfected cells and pNL97-transfected cells) were mixed before lysis. These controls indicate that the trans-splicing products were generated inside the cells, not during the course of the assay. Moreover, trans-spliced products were 
A
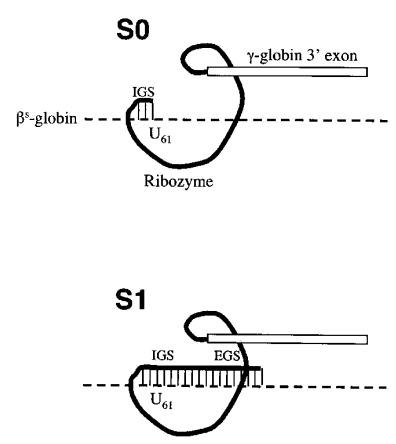
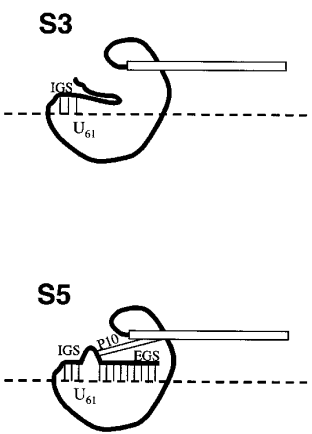

B

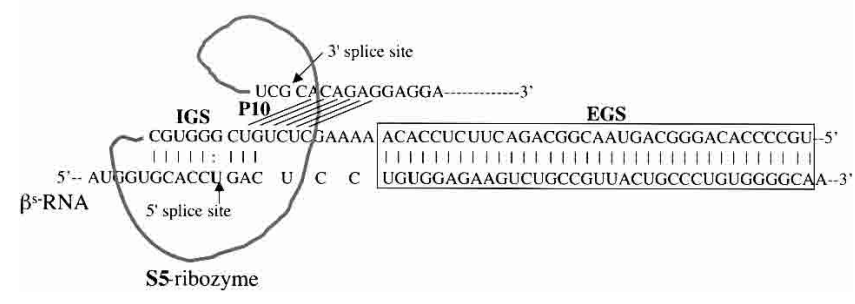

C

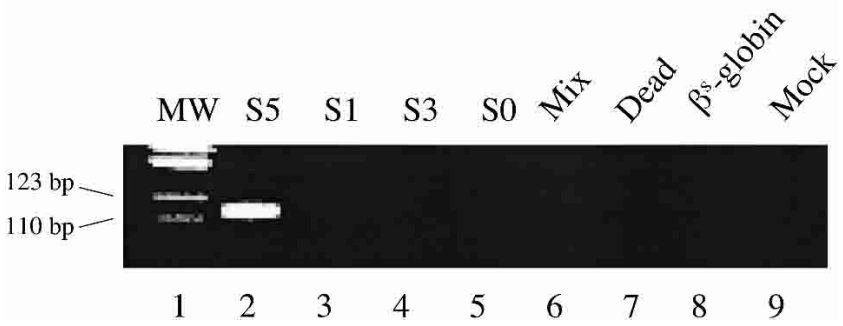

FIGURE 1. Ribozymes derivatives and their activity in mammalian cells. ( $A$ ) Variants of the Rib61-3' $\gamma$ ribozyme shown bound to sickle $\beta$-globin target RNA (dashed line). The S0 ribozyme has a 6-nucleotide internal guide sequence (IGS) that is complementary to the target RNA; the S1 ribozyme has a 6-nucleotide IGS and a 41-nucleotide extended guide sequence (EGS) that is complementary to the target RNA; the S3 ribozyme has a 6-nucleotide IGS and a 41-nucleotide nonspecific spacer sequence at its $5^{\prime}$ end; and the S5 ribozyme has a 9-nucleotide IGS, a 35-nucleotide EGS that is complementary to the target RNA, and a 6-nucleotide P10 that is complementary to the $3^{\prime}$ exon sequence. (B) The S5Rib61-3' $\gamma$ ribozyme bound to the sickle $\beta$-globin target RNA. (C) Detection of trans-splicing products by RT-PCR amplification. HEK293 cells were transfected with the $\beta^{\mathrm{S}}$-globin plasmid (pNL97) alone (lane 8) or in conjunction with plasmids encoding the S5, S1, S3, or S0 ribozymes or an inactive version of the S5 ribozyme (Dead). Total RNA was isolated and trans-splicing products detected by RT-PCR amplification as discussed in Materials and Methods. Amplification of trans-splicing products yield a 116-bp product. Molecular mass markers (MW) of 123 and 110 bp are shown.

not detected in RNA from cells containing the other ribozyme derivatives (Fig. 1C, lanes 3-5). Thus, structural elements in the $\mathrm{S} 5$ ribozyme derivative appear to be important for pol II-mediated expression of active trans-splicing ribozymes in mammalian cells.

\section{Trans-splicing can revise a significant fraction of sickle $\beta$-globin RNA}

Once we identified a pol II-expressed ribozyme (S5) that is active in mammalian cells, we then investigated the efficiency with which this ribozyme could revise sickle $\beta$-globin transcripts. To evaluate trans-splicing efficiency, we inserted a 18-nucleotide sequence into the $\gamma$-globin 3' exon of the S5 ribozyme that is identical to a region of the $\beta$-globin RNA (nucleotides 119-136). This insertion allowed us to use quantitative-competitive (QC) RT/PCR to evaluate reaction efficiency because both trans-spliced products and unreacted $\beta^{s}$-globin substrate RNAs can be coamplified with a single set of PCR primers (Fig. 2A). This modified S5 ribozyme, called S5eff, was cotransfected with the sickle $\beta$-globin expression plasmid into HEK293 cells and total RNA was isolated and analyzed for unspliced substrate RNA and spliced products. As shown in Figure 2B, QC RT/PCR analysis revealed two distinct bands, a 125-bp band corresponding to the unspliced substrate and a 89-bp band corresponding to the trans-spliced product. Moreover, quan- titation of these bands demonstrated that $\sim 9 \%$ or $3 \%$ of the sickle $\beta$-globin target RNA had been converted to product when the ribozyme expression cassette was introduced into cells at a 20-fold or a 2-fold molar excess over the substrate construct, respectively (Fig. 2B).

To determine whether the S5 ribozyme would be active when expressed from other promoters, we explored the use of the human U1 and the T7 RNA polymerase promoter. Therefore, the CMV promoter in the S5eff plasmid was replaced with these promoters to generate the U1S5eff and T7S5eff constructs. When the trans-splicing efficiency of these constructs was compared to S5eff in cotransfection experiments, we found that U1S5eff yielded slightly reduced but still significant level of trans-splicing activity (Fig. 2C). Strikingly, however, ribozymes expressed from the T7S5eff construct reacted with $>50 \%$ of the targeted globin transcripts inside the cells (Fig. 2C). In these studies, the T7 RNA polymerase-expressed ribozymes accumulate to significantly higher levels ( 25 -fold more) than the ribozyme expressed from the CMV promoter (M. Long, unpubl.). Therefore, to determine whether such a high expression level accounts for the enhanced level of RNA repair achieved using the T7-based ribozyme expression cassette, we altered the T7 promoter transcription start site making it suboptimal for RNA expression. This derivative, called T7S5eff-lo, expresses only $1 \%-2 \%$ as much ribozyme as the parental T7S5eff construct (M. Long, unpubl.). As shown in 
A
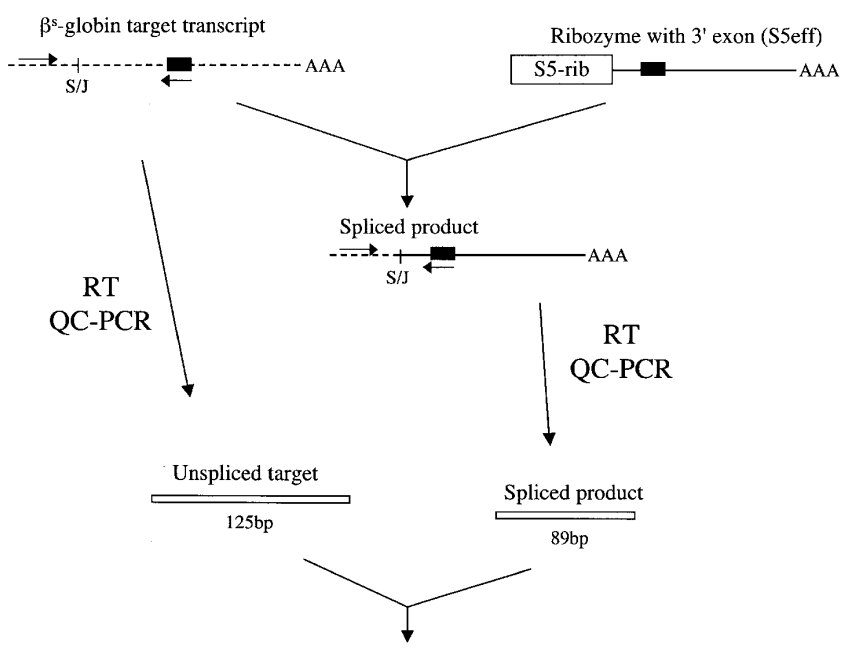

Labeling \& Separation on PAGE
B

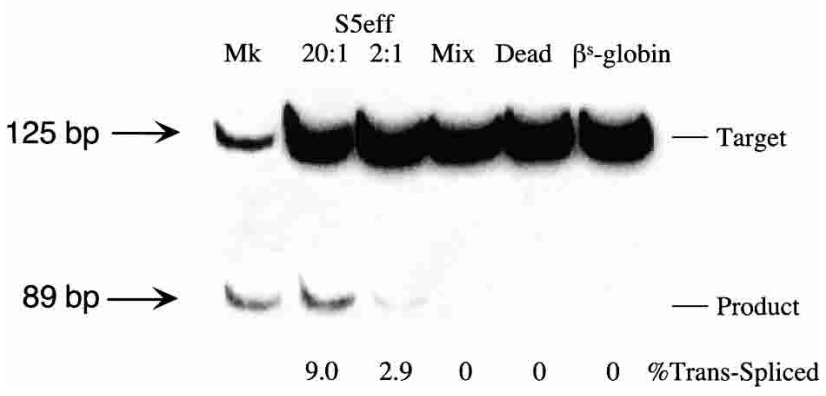

C

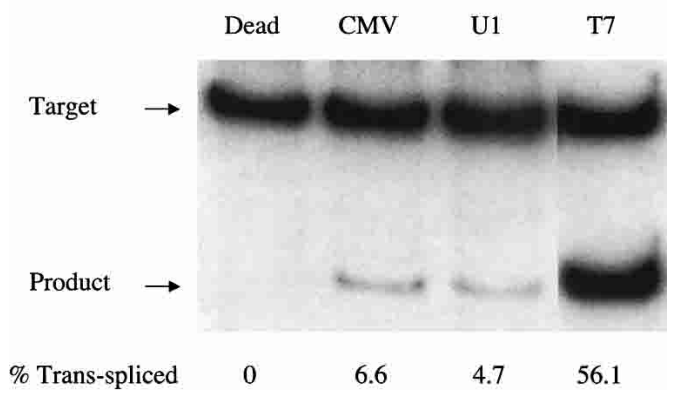

D

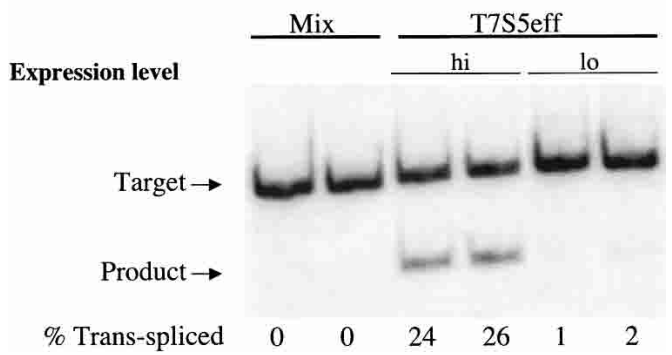

FIGURE 2. Evaluating trans-splicing efficiency of the S5 ribozyme. (A) Scheme for the measurement of trans-splicing efficiency by QC RT-PCR. The sickle $\beta$-globin target RNA (dashed line) and trans-spliced product RNA can be reverse transcribed and coamplified by a single set of PCR primers (arrows) to yield products that differ in size by $36 \mathrm{bp}$. The shaded box denotes the 18 -nucleotide long priming sequence that is present in both the $\beta^{\mathrm{S}}$-globin target RNA (nucleotides 119-136) and the S5eff ribozyme's 3' exon. S/J indicates the trans-splicing junction. (B) The S5eff ribozyme can revise a significant fraction of the sickle $\beta$-globin RNA. HEK293 cells were transfected with the $\beta^{\mathrm{S}}$-globin plasmid alone ( $\beta^{\mathrm{S}}$-globin), or in conjunction with the plasmid expressing the S5eff ribozyme, present in 20-fold (20:1) or 2-fold (2:1) molar excess. Cells independently transfected with $\beta^{\mathrm{S}}$-globin and S5eff plasmids were combined before cell lysis and RNA analysis (Mix). An inactive version of the S5eff ribozyme was cotransfected with the $\beta^{\mathrm{S}}$-globin plasmid (Dead). A mixture of radiolabeled fragments corresponding to the amplified target and product were used as size markers $(\mathrm{Mk})$. (C) The S5eff ribozyme can revise significant fractions of sickle $\beta$-globin RNA when expressed from different promoters. The efficiency of trans-splicing was quantitated in HEK293 cells cotransfected with the $\beta^{S}$-globin plasmid (pNL97) and a 10-fold molar excess of the S5eff ribozyme expression cassettes that are driven by the CMV (S5eff), U1 (U1S5eff), or T7 (T7S5eff) promoters. (D) Effect of varying ribozyme T7 expression levels on repair efficiency. The S5eff ribozyme was transcribed from the T7 promoter in HEK293 cells expressing T7 RNA polymerase. The ribozyme and sickle $\beta$-globin expression cassettes were cotransfected into cells at equal molar amounts. Differences in the transcription start sites in the T7-ribozyme templates result in 100 -fold greater expression of the ribozyme in "hi" (T7S5eff) versus "lo" (T7S5eff-lo) transfected cells. Cells independently expressing ribozyme and substrate RNAs were mixed at the time of cell lysis to generate the "mix" control. Repair efficiency was quantitated for duplicate samples by QC RT-PCR and is reported below the lanes.

Figure 2D, RNA repair is greatly reduced in cells transfected with T7S5eff-lo, indicating that the high level of ribozyme expression from the T7S5eff construct is important for achieving high levels of RNA repair. These results indicate that the $\mathrm{S} 5$ ribozyme is modular and can be expressed from a variety of promoters and retain activity, and moreover they demonstrate that the S5 ribozyme can amend a sig- nificant fraction of sickle $\beta$-globin target RNA when it is expressed at high levels.

\section{Substrate recognition-requirement for additional base-pairing}

One concern about the therapeutic application of transsplicing ribozymes is that they may incorrectly react with 
unintended RNAs inside cells if only minimal base-pairing is required for them to bind target RNAs in this setting (Jones et al. 1996). To investigate the number of base-pairs required for a pol II-expressed ribozyme to recognize its target RNA inside a cell, we generated a series of ribozymes derived from S5eff to systematically analyze the importance of the length of the EGS and the P10 interaction (Fig. 3A). Ribozymes with EGSs that can form of 0 (S6eff), 15 (S9eff), or 54 (S8eff) bp with the sickle $\beta$-globin target RNA (Fig. $3 \mathrm{~A}$ ) were created and compared to the S5eff ribozyme (EGS forms $35 \mathrm{bp}$ ) in cotransfection experiments. As shown in Figure $3 \mathrm{~B}$, the three ribozyme derivatives containing EGSs that form base-pairs with the sickle $\beta$-globin target RNA (S5eff, S8eff, and S9eff) were all able to perform transsplicing in transfected cells to a similar extent. In contrast, no trans-splicing was detected in cells transfected with the S6eff ribozyme, which cannot form an EGS-substrate interaction (Fig. 3B). These results indicate that for pol IIexpressed trans-splicing ribozymes to be active in vivo they require an EGS to form additional base-pairs with their target RNA. To directly determine whether the P10 interaction is also important for trans-splicing activity, we generated and analyzed a S5eff derivative called S10eff in which P10 base-pairing has been disrupted (Fig. 3A). As shown in Figure 3B, no trans-splicing activity was detected in cells expressing the S10eff ribozyme, suggesting that P10 basepairing is critical for activity in vivo.

Although the observation that additional base-pairing between a trans-splicing ribozyme and its target RNA is re- quired for substrate recognition is encouraging with regard to substrate specificity in vivo; the need for such base-pairing also raises concerns because ribozymes with EGSs may engender the degradation of target RNAs by antisense or RNA interference (RNAi)-mediated mechanisms or through activation of the interferon response. To determine whether the extent of base-pairing between the EGS and the sickle $\beta$-globin target RNA could be reduced to alleviate these concerns, we evaluated the ability of the S5eff ribozyme to react with a series of $\beta$-globin RNA derivatives. To this end, 5-bp modular mismatches were introduced cumulatively into the region of sickle $\beta$-globin substrate RNA that is complementary to the external guide sequence of S5eff (Fig. $4 \mathrm{~A})$. As shown in Figure 4B, the S5eff ribozyme can transsplice with these mismatched sickle $\beta$-globin RNA substrates, even when the length of EGS-substrate interaction was shortened to $5 \mathrm{bp}$ without reducing trans-splicing efficiency. Similar results were obtained with the U1S5eff construct where the trans-splicing ribozyme is expressed by the human U1 promoter (data not shown). Thus, the RNA duplex formed between the ribozyme's EGS and the substrate RNA can be shorted to $5 \mathrm{bp}$, a length that is not expected to induce degradation of the target RNA by RNAi or antisense RNA mechanisms (Elbashir et al. 2001). However, removal of the EGS-substrate interaction renders the ribozyme inactive (Figs. 1A, 3A; N. Lan and B. Sullenger, unpubl.).

The observation that a 5-bp EGS-substrate RNA interaction allows trans-splicing to proceed, encouraged us to examine whether a trans-splicing ribozyme could be gener-
A

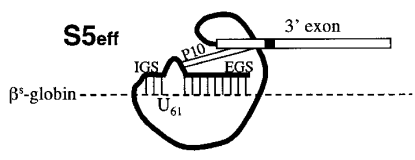

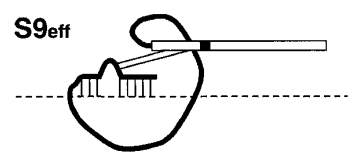

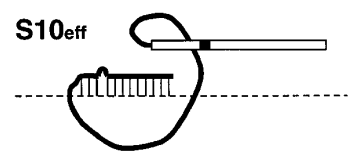

B

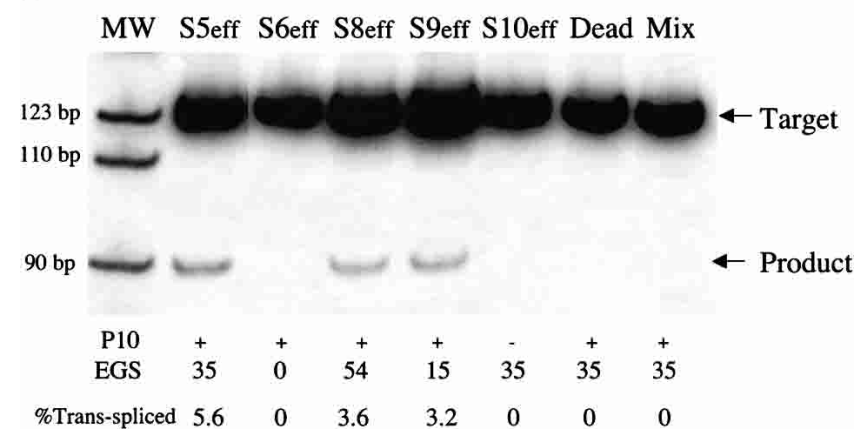

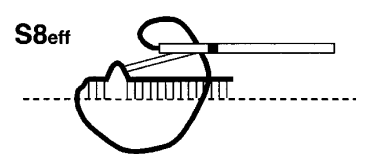

FIGURE 3. Evaluating the importance of the P10 and EGS-target interactions for trans-splicing $(A)$ Derivatives of the S5eff ribozyme with modified P10 or EGS sequences. Ribozyme derivatives pairing to the $\beta^{\mathrm{S}}$-globin target RNA (dashed line) are shown. The shaded box present in the ribozyme 3' exon represents the priming site used to quantitate trans-splicing efficiency by QC RT-PCR. Compared to the 35-bp interaction between the external guide sequence (EGS) of the S5eff ribozyme and the target RNA, S6eff, S9eff, and S8eff ribozymes include an EGS that can form $0 \mathrm{bp}, 15 \mathrm{bp}$, and $54 \mathrm{bp}$, respectively. Although the EGS is unmodified in S10eff, the P10 helix is disrupted. (B) Trans-splicing requires P10 and EGS pairing interactions. Expression constructs encoding the ribozymes described in $(A)$ were co-transfected in 20 -fold molar excess with $\beta^{\mathrm{S}}$-globin plasmid. The percentage of trans-spliced products were determined as described in Figure 2 . For each ribozyme construct, the presence $(+)$ or absence $(-)$ of P10, and the length of the EGS interaction in base-pairs is indicated. Control transfections ("Dead" and "Mix") were performed as in Figure 2B. Molecular mass markers (MW) are shown. 
A

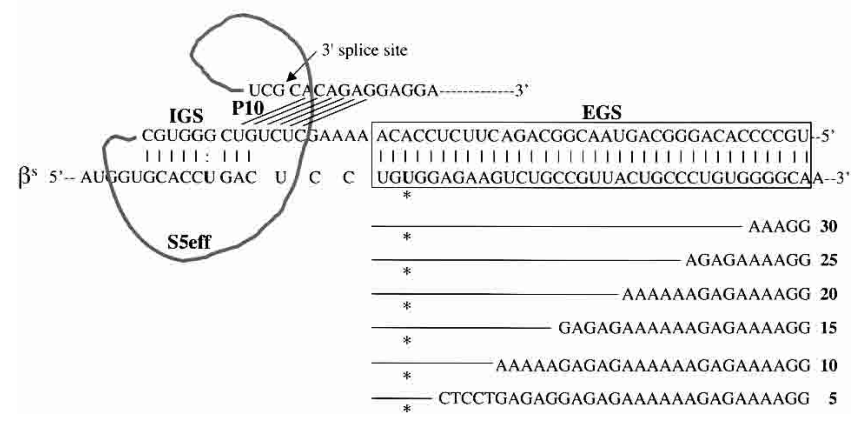

B

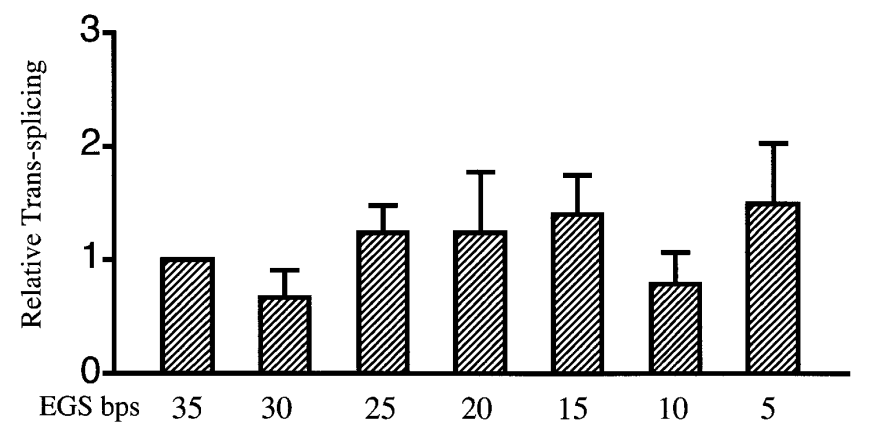

FIGURE 4. Evaluating EGS-target RNA base-pairing requirements. (A) Modifications to the $\beta^{\mathrm{S}}$-globin target sequence. The interaction between the S5eff ribozyme and the $\beta^{\mathrm{S}}$-globin target RNA is shown in detail. The EGS-target interaction is highlighted in the boxed region. Derivatives of $\beta^{\mathrm{S}}$-globin target RNA are represented below the boxed region. Modular mismatches of $5 \mathrm{bp}$ were cumulatively introduced into the target to disrupt increasing regions of the EGS-target interaction. Unaltered regions of the target sequence are represented by the lines. The nucleotide sequences of the mismatched regions in the target RNA are shown. The number of base-pairs remaining in the EGS-target interaction is indicated in bold type. The sickle mutation is denoted with an asterisk. (B) Relative trans-splicing efficiency of the S5eff ribozyme with target RNAs containing mismatches in the region complementary to the ribozyme EGS. The matched (35 EGS bp) and mismatched (5-30 EGS bp) target RNAs were co-expressed with the S5eff ribozyme in HEK293 cells. Trans-splicing efficiency was quantitated by QC RT-PCR analysis for multiple independent transfection experiments $(\mathrm{n}=6)$. Efficiency of trans-splicing was compared to the efficiency of S5eff trans-splicing with the matched $\beta^{\mathrm{S}}$-globin target.

ated that can preferentially react with wild-type $\beta$-globin RNA over sickle $\beta$-globin RNA. Toward this end, the EGS on the S5eff ribozyme was shortened so that it could form only 5 bp with sickle $\beta$-globin RNA. In contrast, this ribozyme called S5eff-5AS can only form 4 bp between its EGS and the wild-type $\beta$-globin RNA (Fig. 5A). The ability of the S5eff-5AS ribozyme and the parental S5eff ribozyme to trans-splice with sickle $\beta$-globin RNA and wild-type $\beta$-globin RNA was assessed. As shown in Figure 5B,C, the S5eff5AS ribozyme reacted significantly better with the sickle $\beta$-globin RNA (6.4\% trans-splicing efficiency) than with the wild-type $\beta$-globin RNA ( $2.2 \%$ efficiency). In contrast the S5eff ribozyme with its 35-nucleotide long EGS reacts equally well with sickle and wild-type $\beta$-globin RNAs (5.0\% and $4.9 \%$ efficiencies, respectively). This result indicates that trans-splicing ribozymes can be generated with short external guide sequences that can at least partially distinguish between closely related substrate RNAs that differ by as little as a single nucleotide.

\section{DISCUSSION}

In this study, we describe the development of trans-splicing ribozyme derivatives that can repair sickle $\beta$-globin RNAs with high efficiency and specificity after gene transfer into and expression in mammalian cells. Surprisingly and in contrast to our previous RNA transfection studies (Lan et al. 1998, 2000), we observed that trans-splicing ribozyme derivatives with 6-nucleotide long IGS were not active in mammalian cells after expression by RNA polymerase II (Fig. 1). However, functional trans-splicing ribozymes could be generated by pol II when the ribozyme was modified to contain a 9-nucleotide IGS, a P10 interaction, and an
EGS (Fig. 1). Moreover, such trans-splicing ribozymes can repair a clinically relevant fraction of sickle $\beta$-globin RNAs in transfected cells when expressed from a variety of promoters (Fig. 2). For repair to occur, the ribozyme must be able to form the P10 interaction with its $\gamma$-globin-derived $3^{\prime}$ exon sequence as well as form at least 5 base pairs between its EGS and the sickle $\beta$-globin substrate RNA (Figs. 3 and 4). Finally, a trans-splicing ribozyme with a 9-nucleotide long IGS and a 5-nucleotide long EGS preferentially reacts with sickle $\beta$-globin RNA over wild-type $\beta$-globin transcripts in cells, although the wild-type RNA only differs from the mutant target transcript by a single nucleotide (Fig. 5). Results from these studies should aid the development of more specific and efficient trans-splicing ribozymes and their corresponding expression cassettes for a variety of RNA repair applications.

A particularly interesting observation is that for a pol II-expressed group I ribozyme to perform trans-splicing in mammalian cells then it must form a P10 interaction with its $3^{\prime}$ exon and base-pair with its target RNA through an EGS. Neither of these interactions are essential for transsplicing to proceed in vitro (Zarrinkar and Sullenger 1998) or after direct transfection of an in vitro transcribed ribozyme into mammalian cells (Lan et al. 1998, 2000). However, Kohler and colleagues (1999) previously demonstrated that such modifications to trans-splicing group I ribozymes greatly enhance the specificity and efficiency of ribozymemediated repair of target RNAs in Escherichia coli. It is currently unclear why these additional sequence elements would be essential for pol II-expressed ribozymes to be active but not be required for ribozymes that are synthesized in vitro and directly transfected into mammalian cells. The P10 interaction is thought to facilitate $3^{\prime}$ exon posi- 
A

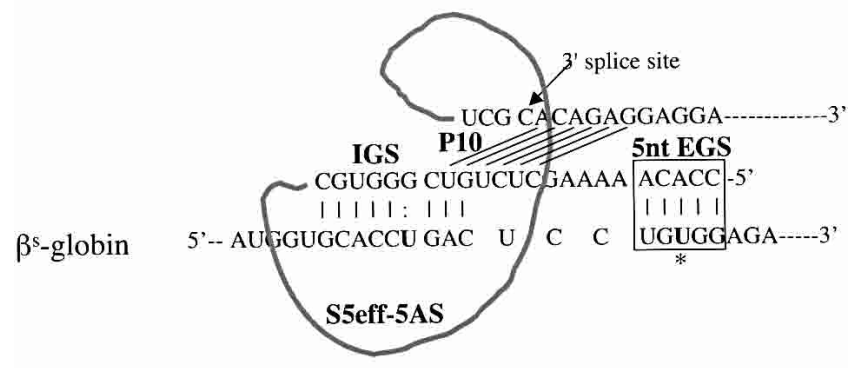

$\beta^{\text {wt }}$-globin $\quad 5^{\prime}$-- AUGGU GCACCU GAC U C C C UGAGGAGA--3'
B

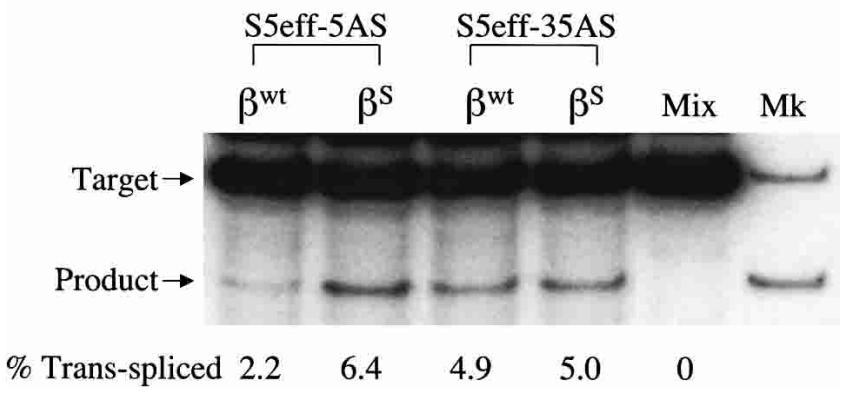

C

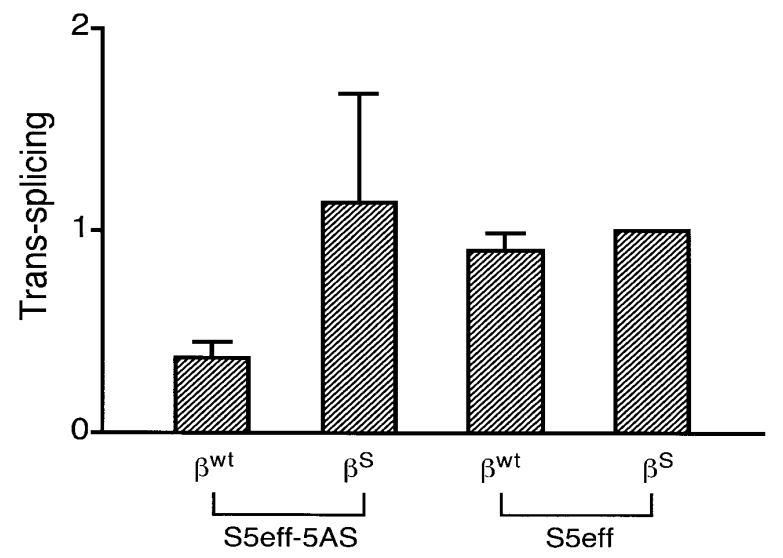

FIGURE 5. A trans-splicing ribozyme that preferentially reacts with sickle $\beta$-globin versus wild-type $\beta$-globin RNA. $(A)$ Design of the S5eff-5AS ribozyme. The interaction between the S5eff-5AS ribozyme and the $\beta^{\mathrm{S}}$-globin target RNA is shown in detail. The EGS-target interaction is limited to $5 \mathrm{bp}$ and is highlighted in the boxed region. The sickle mutation is denoted by the asterisk. The sequence of wild-type $\beta$-globin RNA $\left(\beta^{\mathrm{WT}}\right.$-globin) is shown below for comparison. The wild-type nucleotide corresponding to the sickle mutation is circled. (B) Trans-splicing analysis of S5eff-5AS and S5eff ribozymes with wild-type and sickle $\beta$-globin RNA. Plasmids encoding S5eff ribozymes harboring an EGS of 5 bp (S5eff-5AS) or $35 \mathrm{bp}$ (S5eff-35AS) were cotransfected in twofold molar excess with wild-type $\left(\beta^{\mathrm{WT}}\right)$ or sickle $\left(\beta^{\mathrm{S}}\right) \beta$-globin plasmid. Trans-splicing analysis was carried out as described in Figure 2. Cells independently transfected with $\beta^{\mathrm{S}}$-globin and S5eff plasmids were combined before cell lysis and RNA analysis (Mix). A mixture of radiolabeled fragments corresponding to the amplified target and product were used as size markers (Mk). (C) Comparison of trans-splicing efficiency between the S5eff-5AS and the S5eff ribozyme on wild type $\beta$-globin and sickle $\beta$-globin targets. Independent transfection experiments $(\mathrm{n}=4)$ were analyzed for trans-splicing efficiency as in $(B)$. The results are represented relative to trans-splicing of S5eff on $\beta^{\mathrm{S}}$-globin target RNA.

tioning for the second step of splicing (Suh and Waring 1990) and the base-pairing formed between the EGS and substrate RNA would be expected to greatly increase ribozyme-binding affinity to its target transcript. Therefore, one plausible explanation may be that these two sequence elements are required to stabilize the active conformation of the ribozyme in the face of heteronuclear ribonuclear proteins (hnRNPs) that load onto pol II-generated transcripts. Previously, two groups of investigators reported that hnRNP Al can enhance or inhibit hammerhead ribozyme activity in vitro depending on the concentration of the hnRNP and the length of the binding arms of the ribozymes (Bertrand and Rossi 1994; Herschlag et al. 1994). For example, Bertrand and Rossi (1994) demonstrated that hnRNP A1 inhibits the activity of hammerhead ribozymes that form $<12 \mathrm{bp}$ with their substrate RNAs but did not affect cleavage of substrates bound by 13 or $14 \mathrm{bp}$. This observation is particularly intriguing when considered together with the results described herein that pol II-expressed group I ribozymes need to form at least $14 \mathrm{bp}$ with their target RNAs (9 bp from P1 and 5 bp from the EGS) to perform trans-splicing in mammalian cells.

The observation that pol II-expressed trans-splicing ribozymes require an additional base-pairing interaction with their target RNAs suggests that ribozyme-mediated RNA repair can be made to be much more specific in mammalian cells than previously anticipated from experiments using $\mathrm{T} 7$ RNA polymerase to express such ribozymes (Jones et al. 1996). In contrast to these previous T7 studies, group I ribozymes expressed by RNA pol II do not appear to be able to perform trans-splicing in mammalian cells when they contain an IGS that can form only 6 bp with their target 
RNA (Figs. 1 and 3). Rather, pol II-derived ribozymes appear to also require an EGS that forms at least 5 additional bp with their target transcript (Figs. 3 and 4). This additional base-pairing requirement is anticipated to greatly increase trans-splicing ribozyme specificity for target RNAs because a 14-nucleotide target sequence is much less common in the expressed RNA genome than a 6-nucleotide target sequence. Although the EGS target RNA interaction can be much longer, it does not appear to have to be more than 5 bp in length (Figs. 3 and 4). A significant advantage of being able to make the EGS-target duplex rather short (5 bp) is that such ribozyme binding to its substrate should not induce RNAi activity in ribozyme-expressing cells and inadvertently induce the degradation of the target RNA and ribozyme. Moreover, as previously hypothesized by Herschlag (1991), a ribozyme with a modest EGS-target RNA interaction $(5 \mathrm{bp})$ can distinguish between closely related RNAs such as the wild-type and sickle $\beta$-globin transcripts that differ by only a single nucleotide, whereas a ribozyme with a longer EGS-target RNA interaction (35 bp) cannot distinguish between such substrate RNAs (Fig. 5).

In these studies, the trans-splicing ribozymes were able to repair a clinically relevant fraction of the sickle $\beta$-globin RNA in transfected cells. The trans-splicing efficiency we routinely observed with the S5eff and S5eff-5AS ribozymes was $\sim 10 \%$ after pol II expression and $>50 \%$ with T7 expression of the ribozyme (Figs. 2 and 3). This level of repair using a ribozyme with a $\gamma$-globin $3^{\prime}$ exon would be significant for the treatment of sickle cell anemia, because the most serious manifestations of the disease could be eliminated by expression of $10 \%$ fetal hemoglobin (Powars et al. 1984). We are now constructing lentivirus-based vectors to deliver and express these optimized trans-splicing ribozyme constructs in MEL cell lines and in mouse models of sickle cell disease. Results from these studies will allow us to determine whether similar levels of RNA repair can be achieved in these settings after gene transfer as well as determine whether such levels of RNA repair can result in phenotypic correction of the sickle cell phenotype in vivo.

\section{MATERIALS AND METHODS}

\section{Plasmids}

The pCS0R61-3' $\gamma$ (S0) was derived from pT7R61G (Lan et al. 2000) by replacing T7 promoter with the HCMV promoter and inserting a SV40 polyadenylation signal. Rib61-3' $\gamma$ was placed immediately downstream of the HCMV immediate early promoter. The HCMV promoter sequence was amplified from pMP6 (Applied Immune Sciences Inc.) and inserted between the EcoRI and SphI sites of pT7R61G. The SV40 polyadenylation signal sequence was amplified from pEGFP-N1 (Clontech) and inserted between the EagI and ApaI sites of pT7R61G. The pCS1R61-3' $\gamma$ (S1), pCS3R61-3' $\gamma$ (S3), and pCS5R61-3' $\gamma$ (S5) were derived from the S0 using the following oligonucleotide sets. After annealing, they were inserted between SacI and SphI sites of the S0. (For S1, 5'-CGT TTAGTGAACCGTGCCCCACAGGGCAGTAACGGCAG ACT TCTCCACAGGAGTCGGGTGCAAAAGTTATCAGGCATG and 5 '-CCTGATAACTTTTGCACCCGACTCCTGTGGAGAAGT CTGCCGT TACTGCCCTGTGGGGCACGGT TCACTAAACGAG CT. For S3, 5'-CGTTTAGTGAACCGTGAAAAAGAAAAAAGAA GAAAAAAAAGAAAAAAAGAAAAAAGGGTGCAAAAG TTATC AGGCATG and 5'-CCTGATAACTTTTGCACCCTTTTTTCTTT TTTTCTTTTTTTTCTTCTTTTTTCTTTTTCACGGTTCACTAA ACGAGCT. For S5, 5' -CGTTTAGTGAACCGTGCCCCACAGGG CAGTAACGGCAGACT TCTCCACAAAGAACTCTGTCGGGTG CAAAAGTTATCAGGCATG and 5'-CCTGATAACTTTTGCAC CCGACAGAGTTGTT TGTGGAGAAGTCTGCCGTTACTGCCC TGTGGGGCACGGTTCACTAAACGAGCT. Antisense regions are shown in bold.)

pCS5R61-3' $\gamma$ eff (S5eff) was derived from S5 by overlapping PCR. Two PCR reactions were performed using S5 as a template to generate the two halves of R61-3' eff (first half: upstream primer 5'-GTTATCAGGCATGCACCTGG; downstream primer 5'-CCC CACAGGCTTGTGATAAGGGCCTCACCACCAACTGTAGCCT TGTCCTCCTCT; second half: upstream primer 5'-AGAGGAG GACAAGGCTACAGTTGGTGGTGAGGCCCTTATCACAAGCC TGTGGGG; downstream primer 5'-GGGCGGCCGTCAGTGGT ATCTGGAGGAC). Then the products from the two PCR reactions were mixed as templates for overlapping PCR (upstream primer 5'-GTTATCAGGCATGCACCTGG; downstream primer 5'-GGGCGGCCGTCAGTGGTATCTGGAGGAC). The PCR product (R61-3' $\gamma$ eff) was cloned into SphI and EagI sites of S5 to give S5eff. The S6eff, S9eff, and S10eff were derived from the S5eff by oligonucleotide cloning. Oligonucleotides containing EGS were inserted between SacI and SphI sites of S5eff. (For S6eff, 5' -CGTT TAGTGAACCGTGAAAAAGAAAAAAGAAGAAAAAAAAGAAA AAAAGAAAAACTCTGTCGGGTGCAAAAGTTATCAGGCATG and 5'-CCTGATAACTTTTGCACCCGACAGAGTTTTTCTTTT TTTCTTTTTTTTCTTCTTTTTTCTTTTTCACGGTTCACTAAA CGAGCT. For S9eff, 5'-CGTTTAGTGAACCGTGCAGACTTC TCCACAAAGAACTCTGTCGGGTGCAAAAGTTATCAGGCATG and 5'-CCTGATAACTTTTGCACCCGACAGAGTTGTTTGTGG AGAAGTCTGCACGGTTCACTAAACGAGCT. For S10eff, 5' CGTTTAGTGAACCGTGCCCCACAGGGCAGTAACGGCAGAC T TCTCCACAGAAACAAACGTCGGGTGCAAAAGT TATCAGG CATG and 5'-CCTGATAACTTTTGCACCCGACGTTTGTTTC TGTGGAGAAGTCTGCCGTTACTGCCCTGTGGGGCACGGTT CACTAAACGAGCT. Antisense regions are shown in bold.) The S8eff was derived from S5eff using the following PCR primers (upstream primer 5'-CGTTTAGTGAACCGTGACTTCATCCAC GTTCACCTTGCCCCACAGGGCAGTAACG; downstream primer 5'-GGGCGGCCGTCAGTGGTATCTGGAGGAC) followed by cloning into SacI and EagI sites of S5eff. The S5eff-d (Dead) construct has a 98-nucleotide deletion between BglII and NheI sites of Rib61 and served as an inactive control for ribozyme activity.

To generate substrate plasmids (pNL97 and pNL65), the $\beta^{\text {s }}$ globin and wild-type $\beta$-globin genomic DNAs were amplified from pTR97 and pTR65 (generous gifts from Dr. Townes, University of Alabama at Birmingham). The PCR product with NheI/ $B g l \mathrm{II}$ ends was inserted between NheI and BamHI sites of pMP6 (Applied Immune Sciences Inc.). To exclude any mutations from PCR, the inner region was replaced with the original fragment from pTR97 and pTR65 using BspMI and SwaI sites followed by verification of the sequences of the two ends. 
Human U1 promoter was amplified from pBlskU1pr (a generous gift from Dr. Rossi, Beckman Research Institute of the City of Hope) using the primers 5'-GTCGACTAAGGACCAGCTTC-3' and $5^{\prime}$-GCATGCCTGATAACTTTTGCACCCGACAGAGTTCTT TGTGGAGAAGTCTGCCGTTACTGCCCTGTGGGGCAGATGA TCTTCGGGC. The resulting fragment was cloned into SalI/SphI sites of the S5eff to generate U1S5eff. Human $\beta$-globin promoter and optimized version of human $\beta$-globin promoter were amplified from pTR65 with the following primer sets: 5'-GTCGACG CATGCATGAGCAA- ${ }^{\prime}$ ' and $5^{\prime}$-GCATGCCTGATAACTTTTGCA CCCGACAGAGTTCTTTGTGGAGAAGTCTGCCGTTACTGCC CTGTGGGGCACCATGGTGTCTGTT for human $\beta$-globin promoter and 5'-GTCGACGTACGGCTGTCATC-3' and 5'-GCAT GCCTGATAACTTTTGCACCCGACAGAGTTCTTTGTGGAGAA GTCTGCCGTTACTGCCCTGTGGGGCACCATGGTGTCTGTT for optimized version of the human $\beta$-globin promoter. The resulting fragments were inserted into SalI/SphI sites of the S5eff to obtain $\beta$ S5eff and OßS5eff.

Mutant substrate plasmids were made using QuikChange SiteDirected Mutagenesis Kit (Stratagene) on pNL97 plasmid. (Only the top primer sequence is shown because the bottom one is complementary to the top one. The region of mutation is marked in bold.) For -1SB: 5' -CACCATGGTGCACCAGACTCCTGTGG AG-3', for -2SB: 5'-CACCATGGTGCACATGACTCCTGTGG3', for -4SB: 5'-GACACCATGGTGCTCCTGACTCCTGTGG-3', for $-6 \mathrm{SB}: 5^{\prime}$-CAGACACCATGGTACACCTGACTCCTGTGG-3', for -2-4SB: 5'-GACACCATGGTGCTCATGACTCCTGTGG-3', for 30SB: 5' -CTGCCCTGTGAAAGGAGGTGAACGTGG, for 25SB: 5'-CCGTTACTGCCAGAGAAAAGGAGGTGAACGTGG, for 20SB: 5'-GTCTGCCGTTAAAAAAAGAGAAAAGGAGGTGAACGTGG3', for 15SB: 5'-GGAGAAGTCTGCGAGAGAAAAAAGAGAAAA GGAGGTGAACGTGG, for 10SB: 5'-GACTCCTGTGGAGAAGA AAAAGAGAGAAAAAAGAGAAAAGGAGGTGAACGTGGATG3', for 5SB: 5'-GACTCCTGTGGCTCCTGAGAGGAGAGAAAAA AGAGAAAAGGAGGTGAACGTGG, for 0SB: 5'-GCACCTGAC TCCAAAAACTCCTGAGAGGAGAGAAAAAAGAGAAAAGGAG GTGAACGTGG.

The S5eff-5AS was constructed using QuikChange Site-Directed Mutagenesis Kit (Stratagene) on the S5eff plasmid. The primers used are 5'-GAGCTCGTTTAGTGAACCGCCACAAAGAACTC TGTCGGG-3' (top) and 5 -'cccgacagagttctttgtggcggttcactaaacgag ctc-3' (bottom).

\section{Cell lines}

HEK293 cells were grown in Dulbecco's modified Eagle's medium supplemented with $10 \%(\mathrm{v} / \mathrm{v})$ fetal bovine serum(GIBCO-BRL) at $37^{\circ} \mathrm{C}$ under $5 \% \mathrm{CO}_{2}$.

\section{Transfection}

HEK293 cells were transfected on 12-well plates using LipofectAMINE 2000 reagents (Life Technologies). The eGFP expression plasmid was transfected in parallel to monitor transfection efficiency, which ranged from $40 \%-70 \%$. The cells were harvested for analysis $24-48 \mathrm{~h}$ after transfection.

\section{QC RT/PCR}

The total RNA was isolated using Trizol reagent (Life Technologies). To inhibit ribozyme activity during RNA preparation, EDTA was added to Trizol before extraction to a final concentration of 20 $\mathrm{mM}$. For mix controls, the ribozyme-transfected cells were combined with the substrate-transfected cells shortly before lysis. The harvested RNAs were treated with RNase-free DNase I (Promega) at $37^{\circ} \mathrm{C}$ for $5 \mathrm{~min}$. The reaction buffer contained $10 \mathrm{mM} \mathrm{L}$-argininamide to competitively inhibit ribozyme activity. The reaction mixture was then extracted with phenol/chloroform, chloroform, and precipitated with absolute ethanol. The RNA was reverse-transcribed at $37^{\circ} \mathrm{C}$ for $15 \mathrm{~min}$ in the presence of $\mathrm{L}$-argininamide $(10 \mathrm{mM})$ with a primer specific for $3^{\prime}$ exon $\left(3^{\prime} \gamma\right.$ globin primer: $5^{\prime}$-CGGGAATTCCCACAGGCTTGTGATA- ${ }^{\prime}$ 'or down-10 primer: 5'-AGGGCCTCACCACCAACT-3').

The cDNAs were then amplified for 30 cycles $\left(94^{\circ} \mathrm{C}\right.$ for $30 \mathrm{sec}$, $64^{\circ} \mathrm{C}$ for $30 \mathrm{sec}, 72^{\circ} \mathrm{C}$ for $1 \mathrm{~min}$ ) using the same $3^{\prime}$ exon primer and a $5^{\prime}$ primer specific for $\beta$-globin ( $5^{\prime}$-GGGGATCCCTGTGT TCACTAGCAACC). For labeling, $3 \mu \mathrm{L}$ of PCR product was used as template for new PCR (5 cycles) in the presence of $\left[\alpha-{ }^{32} \mathrm{P}\right] \mathrm{dCTP}$. The radioactive products were run on $10 \%$ nondenaturing polyacrylamide gel and quantitated using a Storm 860 PhosphorImager and Image Quant Software (Molecular Dynamics).

\section{Trans-splicing analysis of ribozymes expressed by $\mathrm{T} 7$ RNA polymerase}

Trans-splicing ribozyme templates were amplified by PCR from the pCMV-S5eff construct to append a T7 promoter upstream of the ribozyme (T7S5eff). To alter the ribozyme expression levels, two versions of the template were produced: T7S5eff, which contains a favorable transcription start site (gggag), and T7S5-lo, which contains a less favorable transcription start site (cccca). Amplified templates were prepared for transfection by phenol/ chloroform extraction and ethanol precipitation. The precipitated products were purified from an agarose gel and extracted from the gel using a Qiaex II kit. Extracted DNA was resuspended in TrisEDTA (TE) at a concentration of 100-200 ng/ $\mu \mathrm{L}$. Template DNA $(0.1 \mu \mathrm{g})$ was co-transfected with substrate plasmid pNL97 $(2 \mu \mathrm{g})$, together with $0.5 \mu \mathrm{g}$ of the T7 RNA expression plasmid, pCMVT7-T7pol (gift of Dr. L. Huang) into 293 cells. Total RNA was harvested from transfected cells $24 \mathrm{~h}$ after transfection in TriReagent supplemented with $10 \mathrm{mM}$ EDTA. Total RNA was analyzed by QC RT-PCR as described.

\section{ACKNOWLEDGMENTS}

We thank Chris Rusconi and J.P. Jones for critical reading of the manuscript and helpful discussions. This study was supported in part by grants from the Susan B. Komen Foundation (J.B.) and from the National Institutes of Health (B.A.S.).

The publication costs of this article were defrayed in part by payment of page charges. This article must therefore be hereby marked "advertisement" in accordance with 18 USC section 1734 solely to indicate this fact.

Received March 5, 2003; accepted July 24, 2003.

\section{REFERENCES}

Been, M.D. and Cech, T.R. 1986. One binding site determines sequence specificity of Tetrahymena pre-rRNA self-splicing, transsplicing, and RNA enzyme activity. Cell 47: 207-216. 
Bertrand, E.L. and Rossi, J.J. 1994. Facilitation of hammerhead ribozyme catalysis by the nucleocapsid protein of HIV-1 and the heterogeneous nuclear ribonucleoprotein A1. EMBO J. 13: 29042912.

Couture, L.A. and Stinchcomb, D.T. 1996. Anti-gene therapy: The use of ribozymes to inhibit gene function. Trends Genet. 12:510515.

Elbashir, S.M., Harborth, J., Lendeckel, W., Yalcin, A., Weber, K., Tuschl, T. 2001. Duplexes of 21-nucleotide RNAs mediate RNA interference in cultured mammalian cells. Nature 411: 494-498.

Herschlag, D. 1991. Implications of ribozyme kinetics for targeting the cleavage of specific RNA molecules in vivo: More isn't always better. Proc. Natl. Acad. Sci. 88: 6921-6925.

Herschlag, D., Khosla, M., Tsuchihashi, Z., and Karpel, R.L. 1994. An RNA chaperone activity of non-specific RNA binding proteins in hammerhead ribozyme catalysis. EMBO J. 13: 2913-2924.

Jennings, P.A. and Molloy, P.L. 1987. Inhibition of SV40 replicon function by engineered antisense RNA transcribed by RNA polymerase III. EMBO J. 6: 3043-3047.

Jones, J.T., Lee, S.-W., and Sullenger, B.A. 1996. Tagging ribozyme reaction sites to follow trans-splicing in mammalian cells. Nat. Med. 2: 643-648.

Kohler, U., Ayre, B.G., Goodman, H.M., and Haseloff, J. 1999. Transsplicing ribozymes for targeted gene delivery. J. Mol. Biol. 285: $1935-1950$.

Lan, N., Howrey, R.P., Lee, S.-W., Smith, C.A., and Sullenger, B.A. 1998. Ribozyme mediated repair of sickle $\beta$-globin mRNAs in erythrocyte precursors. Science 280: 1593-1596.

Lan, N., Rooney, B.L., Lee, S.-W., Howrey, R.P., Smith, C.A., and Sullenger, B.A. 2000. Enhancing RNA repair efficiency by combining trans-splicing ribozymes that recognizes different accessible sites on target RNA. Mol. Ther. 2: 245-255.

Lee, N.S., Dohjima, T., Bauer, G., Li, H., Li, M.J., Ehsani, A., Salvaterra, P., Rossi, J. 2002. Expression of small interfering RNAs targeted against HIV-1 rev transcripts in human cells. Nat. Biotechnol. 19: $500-505$.

Lehnert, V., Jaeger, L., Michel, F., and Westhof, E. 1996. New looploop tertiary interactions in self-splicing introns subgroup IC and ID: A complete 3D model of the Tetrahymena thermophila ribozyme. Chem. Biol. 3: 993-1009.

Michel, F. and Westhof, E. 1990. Modelling of the three-dimensional architecture of group I catalytic introns based on comparative sequence analysis. J. Mol. Biol. 216: 585-610.

Michel, F., Hanna, M., Green, R., Bartel, D.P., and Szostak, J.W. 1989. The guanosine binding site of the Tetrahymena ribozyme. Nature
342: 391-395.

Miyagishi, M. and Taira, K. 2002. U6 promoter-driven siRNA with four uridine $3^{\prime}$ overhangs efficiently suppress targeted gene expression in mammalian cells. Nat. Bio. 19: 497-500.

Ojwang, J.O., Hampel, A., Looney, D.J., Wong-Staal, F., and Rappaport, J. 1992. Inhibition of human immunodeficiency virus type 1 expression by a hairpin ribozyme. Proc. Natl. Acad. Sci. 89: 1080210806.

Paul, C.P., Good, P.D., Winer, I., Engelke, D.R. 2002. Effective expression of small interfering RNA in human cells. Nat. Biotechnol. 19: 505-508.

Phylactou, L.A., Kilpatrick, M.W., and Wood, M.J. 1998. Ribozymes as therapeutic tools for genetic disease. Hum. Mol. Genet. 7: 16491653.

Powars, D.R., Weiss, J.N., Chan, L.S., and Schroeder, W.A. 1984. Is there a threshold level of fetal hemoglobin that ameliorates morbidity in sickle cell anemia? Blood 63: 921-926.

Shatkin, A.J. 1985. mRNA cap binding protein: Essential factors for initiating translation. Cell 40: 223-224.

Suh, E.R. and Waring, R.B. 1990. Base pairing between the $3^{\prime}$ exon and an internal guide sequence increases $3^{\prime}$ splice site specificity in the Tetrahymena self-splicing rRNA intron. Mol. Cell. Biol. 10: 29602965.

Sui, G., Soohoo, C., Affar el, B., Gay, F., Shi, Y., Forrester, W.C., Shi, Y. 2002. A DNA vector-based RNAi technology to suppress gene expression in mammalian cells. Proc. Natl. Acad. Sci. 99: 55155520.

Sullenger, B.A. and Cech, T.R. 1994. Ribozyme-mediated repair of defective mRNA by targeted trans-splicing. Nature 317: 619-622.

Sullenger, B.A., Lee, T.C., Smith, C.A., Ungers, G.E., and Gilboa, E. 1990a. Expression of chimeric tRNA-antisense transcripts renders NIH 3T3 cells highly resistant to Moloney murine leukemia virus replication. Mol. Cell. Biol. 10: 6512-6523.

Sullenger, B.A., Gallardo, H.F., Ungers, G.E., and Gilboa, E. 1990b. Overexpression of TAR sequences renders cells resistant to human immunodeficiency virus replication. Cell 63: 601-608.

Waring, R.B., Towner, P., Minter, S.J. and Davies, R.W. 1986. Splicesite selection by a self-splicing RNA of Tetrahymena. Nature 321: 133-139.

Watanabe, T. and Sullenger, B.A. 2000. Induction of wild-type p53 activity in human cancer cells by ribozymes that repair mutant $p 53$ transcripts. Proc. Natl. Acad. Sci. 97: 8490-8494.

Zarrinkar, P.P. and Sullenger, B.A. 1998. Probing the interplay between the two steps of group I intron splicing: competition of exogenous guanosine and $\omega \mathrm{G}$. Biochemistry 37: 18056-18063. 

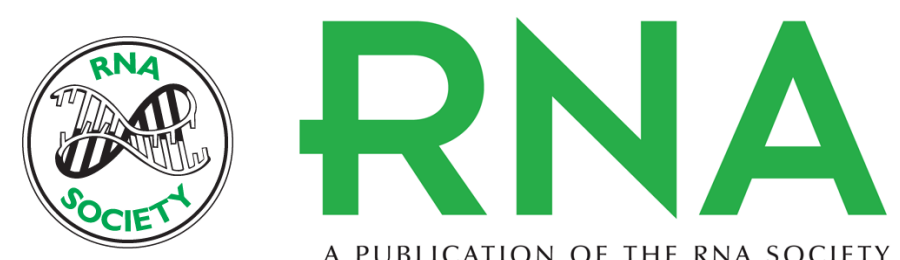

A PUBLICATION OF THE RNA SOCIETY

\section{Efficient and specific repair of sickle $\beta$-globin RNA by trans-splicing ribozymes}

JONGHOE BYUN, NING LAN, MEREDITH LONG, et al.

RNA 2003 9: 1254-1263

References This article cites 29 articles, 8 of which can be accessed free at:

http://rnajournal.cshlp.org/content/9/10/1254.full.html\#ref-list-1

\section{License}

Email Alerting Receive free email alerts when new articles cite this article - sign up in the box at the Service top right corner of the article or click here. 